Optical emission spectroscopy of oxygen plasma induced by $\mathrm{IR}_{2} \mathrm{CO}_{2}$ pulsed laser

This article has been downloaded from IOPscience. Please scroll down to see the full text article.

2008 J. Phys. D: Appl. Phys. 41215206

(http://iopscience.iop.org/0022-3727/41/21/215206)

View the table of contents for this issue, or go to the journal homepage for more

Download details:

IP Address: 161.111.22.141

The article was downloaded on 13/12/2012 at 08:54

Please note that terms and conditions apply. 


\title{
Optical emission spectroscopy of oxygen plasma induced by IR $\mathrm{CO}_{2}$ pulsed laser
}

\author{
J J Camacho ${ }^{1}$, M Santos ${ }^{2}$, L Díaz $^{2}$ and J M L Poyato ${ }^{1}$ \\ ${ }^{1}$ Departamento de Química-Física Aplicada, Facultad de Ciencias, Universidad Autónoma de Madrid, \\ Cantoblanco, 28049-Madrid, Spain \\ ${ }^{2}$ Instituto de Estructura de la Materia, CFMAC, CSIC, Serrano 121, 28006-Madrid, Spain \\ E-mail: j.j.camacho@uam.es
}

Received 4 July 2008, in final form 1 September 2008

Published 14 October 2008

Online at stacks.iop.org/JPhysD/41/215206

\begin{abstract}
Laser-induced breakdown (LIB) spectroscopy in oxygen at room temperature and pressures ranging from 4.6 to $75 \mathrm{kPa}$ was studied using a high-power transverse excitation atmospheric $\mathrm{CO}_{2}$ laser $\left(\lambda=9.621\right.$ and $10.591 \mu \mathrm{m} ; \tau_{\mathrm{FWHM}}=64 \mathrm{~ns}$; power densities ranging from 0.87 to $6.31 \mathrm{GW} \mathrm{cm}^{-2}$ ). The spectrum of the generated plasma is dominated by emission of strong $\mathrm{O}$, $\mathrm{O}^{+}$and weak $\mathrm{O}^{2+}$ atomic lines. Excitation temperatures of $31500 \pm 1600 \mathrm{~K}$ and $23000 \pm 3000 \mathrm{~K}$ were estimated by means of $\mathrm{O}^{2+}$ and $\mathrm{O}^{+}$ionic lines, respectively. Electron number densities of the order of $(3.5-16.5) \times 10^{16} \mathrm{~cm}^{-3}$ were deduced from the Stark broadening of several ionic $\mathrm{O}^{+}$lines. The characteristics of the spectral emission intensities from different species have been investigated as functions of the oxygen pressure and laser irradiance. Optical breakdown threshold intensities in $\mathrm{O}_{2}$ at $10.591 \mu \mathrm{m}$ have been determined. The physical processes leading to LIB of oxygen have been analysed.
\end{abstract}

(Some figures in this article are in colour only in the electronic version)

\section{Introduction}

Oxygen, being the third most abundant element in the universe by mass [1], one of the most abundant constituents in the Earth's atmosphere and the most abundant element by mass in the Earth's crust, is of great interest to many different researchers. It is well known that gases such as $\mathrm{O}_{2}$ under normal absorption conditions are relatively transparent to laser radiation. However, when the laser intensity increases and reaches a certain threshold value they are no longer transparent. The laser-induced breakdown (LIB) is characterized by an intense plasma or spark, brilliant flash and a cracking noise. Measurements of the emission spectra revealed a highly ionized plasma which expanded somewhat like a blast wave into the surrounding medium. Since the first observations of LIB in gases [2], the phenomenon has been studied extensively, both theoretically and experimentally [3-11]. Among the various diagnostic techniques which are convenient tools for detecting various transient species from UV up to IR spectral regions, optical emission spectroscopy (OES) has definite advantages pertaining to high spatial and temporal resolution without perturbation of the laser-induced plasma.
Laser-induced breakdown spectroscopy (LIBS) is a simple spectrochemical technique in which a laser beam is directed at a sample to create a high-temperature plasma. Several reviews on the LIBS principle and applications can be found in [12-18]. Detailed studies of LIBS on $\mathrm{N}_{2}, \mathrm{Si}_{3} \mathrm{H}_{8}$ and graphite have been recently made in our laboratory [19-21]. Though many breakdown measurements have been carried out in air and nitrogen, there is much less data on oxygen. Several laser-induced breakdown threshold measurements have been carried out in oxygen [22-27]. However, very limited data are available on the laser-induced plasma emission spectrum in oxygen $[24,28]$. The LIBS oxygen spectrum recorded by Nordstrom [28] in the spectral region 350-950 $\mathrm{nm}$ is composed of $\mathrm{O}$ and $\mathrm{O}^{+}$atomic lines.

In this work, LIB generated by $\mathrm{CO}_{2}$ laser pulses in $\mathrm{O}_{2}$ has been investigated by means of OES. The plasma induced in oxygen with pressures ranging from 4.6 to $75 \mathrm{kPa}$ has been measured and analysed. Optical breakdown threshold intensities in oxygen at $10.591 \mu \mathrm{m}$ have been measured. The strong emission observed in the plasma region is mainly due to the relaxation of excited atomic $\mathrm{O}$ and ionic fragments $\mathrm{O}^{+}$ and $\mathrm{O}^{2+}$. Excitation temperatures and electron densities were 
calculated by means of different $\mathrm{O}^{2+}$ and $\mathrm{O}^{+}$ionic lines. The physical processes leading to LIB of oxygen in the power density range from 0.87 to $6.31 \mathrm{GW} \mathrm{cm}^{-2}$ have been analysed.

\section{Experimental}

\subsection{Experimental details}

The LIBS system has been described in earlier publications [19-21], hence only a brief overview of the system will be presented. The laser-induced plasma was generated using a transverse excitation atmospheric (TEA) $\mathrm{CO}_{2}$ laser (Lumonics model K-103) operating on an 8:8:84 mixture of $\mathrm{CO}_{2}: \mathrm{N}_{2}: \mathrm{He}$, respectively. The laser is equipped with frontal Ge multimode optics (35\% reflectivity) and a rear diffraction grating with 135 lines $\mathrm{mm}^{-1}$ blazed at $10.6 \mu \mathrm{m}$. The $\mathrm{CO}_{2}$ laser irradiation of the gas was carried out using the $9 \mathrm{P}(28)$ line at $\lambda=9.621 \mu \mathrm{m}(\Delta E=0.1289 \mathrm{eV})$ and the $10 \mathrm{P}(20)$ line at $\lambda=10.591 \mu \mathrm{m}(\Delta E=0.1171 \mathrm{eV})$. These wavelengths were checked with a $16 \mathrm{~A}$ spectrum analyser (Optical Eng. Co.). The pulse temporal profile was monitored with a photon drag detector (Rofin Sinar 7415). The temporal shape of the TEA$\mathrm{CO}_{2}$ laser pulse consisted of a prominent spike of full width at half maximum (FWHM) of 64 ns carrying $90 \%$ of the laser energy, followed by a long lasting tail of lower energy and about $3 \mu$ s duration. The laser pulse repetition rate is usually $1 \mathrm{~Hz}$. The divergence of the emitted laser beam is $3 \mathrm{mrad}$. The pulsed $\mathrm{CO}_{2}$ laser beam was focused with a $\mathrm{NaCl}$ lens of $24 \mathrm{~cm}$ focal length onto the centre of the gas cell. The primary laser beam was angularly defined and attenuated by two diaphragms of 25 and $17.5 \mathrm{~mm}$ diameter before entering the gas cell. The $\mathrm{CO}_{2}$ laser averaged energy was measured in front of the lens with a Lumonics 20D pyroelectric detector through a Tektronix TDS 540 digital oscilloscope. Brewster losses were determined by making pulse energy measurements with and without the $\mathrm{NaCl}$ window in place. The focused radius of the laser beam $(0.499 \mathrm{~mm})$ was measured at the target position with a pyroelectric array Delta Development Mark IV. The laser intensity (power density or irradiance) on the focal volume ranged from 0.86 to $6.31 \mathrm{GW} \mathrm{cm}^{-2}$. The laser power density energy was varied using several calibrated $\mathrm{CaF}_{2}$ attenuators. Experimental threshold power densities for oxygen were measured for the TEA-CO $\mathrm{CO}_{2}$ laser at $\lambda=10.591 \mu \mathrm{m}$. The threshold power densities for oxygen at a given pressure were measured in the following two manners. First the cell was evacuated with the aid of a rotary pump to a base pressure of $4 \mathrm{~Pa}$ that was measured by a mechanical gauge and then it was filled with oxygen up to the desired pressure. The TEA-CO laser was fired and its energy transmitted through the cell was increased until breakdown was observed in 50\% of laser pulses. The threshold was easily determined because it was always associated with the appearance of a blue bright flash of light in the focal region, with a cracking noise, and the abrupt absorption of the laser pulse transmitted through the focal region. Another way to measure the threshold was to induce a previous breakdown at a pressure over the desired value, later the pressure was lowered and the energy adjusted until the breakdown began with some probability, usually around $50 \%$. This method is similar to inducing the breakdown with energy in excess and to attenuating the laser until the spark disappears. The present experiments showed that when high laser energy was used, oxygen breakdown occurred easily and it was reproducible. When the laser energy was reduced to its threshold power density value, oxygen breakdown became a sporadic event. Such sporadic behaviour might be due to the difficulty in generating seed electrons at the breakdown threshold values. The primary $\mathrm{O}_{2}$ studied in these experiments was of research grade purity (>99.995\%). Oxygen gas, initially at ambient temperature ( $298 \mathrm{~K})$, was placed in a cell equipped with a $\mathrm{NaCl}$ window for the laser beam input and two quartz windows for optical access. Optical emission from the plasma plume was collected onto the entrance slit of different monochromators. All the experimental measurements were taken at the focal point at $10 \mathrm{~cm}$ in the direction perpendicular to the plasma symmetry axis. Two spectrometers were used: a 1/8 m Oriel spectrometer ( $25 \mu \mathrm{m}$ slit and grating of 1200 grooves $\mathrm{mm}^{-1}$ ) in the spectral region $2000-10500 \AA$ at a resolution of $\sim 1.3 \AA$ in first order with an Andor DU420-OE (open electrode) CCD camera $\left(1024 \times 256\right.$ matrix of $26 \times 26 \mu \mathrm{m}^{2}$ individual pixels $)$ with thermoelectric cooling working at $-30{ }^{\circ} \mathrm{C}$ and a $0.32 \mathrm{~m}$ ISA Jobin Yvon Spex (Model HR320) spectrometer (adjustable slit and holographic grating of 2400 grooves $\mathrm{mm}^{-1}$ ) in the spectral region $1900-7500 \AA$ at a resolution of $\sim 0.10 \AA$ in first order with an Andor iStar DH734 ICCD camera $(1024 \times 1024$ matrix of $25 \times 25 \mu \mathrm{m}^{2}$ individual pixels) with thermoelectric cooling working at $-20^{\circ} \mathrm{C}$. The low noise level of the ICCD allows long integration times and therefore the detection of very low emission intensities. Many of the spectra were obtained with $5 \mathrm{~s}$ integration time over the entire luminous plasma event. The intensity response of the detection system was calibrated with a standard (Osram No. 6438, 6.6 A, 200 W) halogen lamp and an $\mathrm{Hg} / \mathrm{Ar}$ pencil lamp. Several $(\mathrm{Cu} / \mathrm{Ne}, \mathrm{Fe} / \mathrm{Ne}$ and $\mathrm{Cr} / \mathrm{Ar})$ hollow cathode lamps (HCLs) were used for the spectral wavelength calibration of the spectrometers.

\subsection{Laser parameters for the present experiments}

Since different authors characterize the laser field values in different ways, we give here the relationships between the laser peak power, the peak intensity, the fluence, the photon flux density and the electric field. The laser peak power or radiant pulse energy per time $P_{\mathrm{W}}$ (in $\mathrm{W}$ ) is given by

$$
P_{\mathrm{W}}=E_{\mathrm{W}} / \tau_{\mathrm{FWHM}}
$$

where $E_{\mathrm{W}}$ (in J) is the pulse energy and $\tau_{\mathrm{FWHM}}$ (in s) is the pulse duration at the FWHM. The laser peak intensity (power density or irradiance) $I_{\mathrm{W}}\left(\mathrm{W} \mathrm{cm}^{-2}\right)$, fluence $\Phi_{\mathrm{W}}$ on the focused-spot area $\left(\mathrm{J} \mathrm{cm}^{-2}\right)$, the photon flux $F_{\mathrm{ph}}$ (photon $\mathrm{cm}^{-2} \mathrm{~s}^{-1}$ ) and electric field $F_{\mathrm{E}}\left(\mathrm{V} \mathrm{cm}^{-1}\right)$ are given by

$$
\begin{gathered}
I_{\mathrm{W}}=P_{\mathrm{W}} / \pi r^{2}, \\
\Phi_{\mathrm{W}}=E_{\mathrm{W}} / \pi r^{2}, \\
F_{\mathrm{ph}}=I_{\mathrm{W}} \lambda / h c, \\
F_{\mathrm{E}}=\sqrt{I_{\mathrm{W}} / c \varepsilon_{0}},
\end{gathered}
$$


Table 1. Laser parameters for the present experiments.

\begin{tabular}{lllllll}
\hline $\begin{array}{l}\text { Laser wavelength } \\
(\mu \mathrm{m})\end{array}$ & $\begin{array}{l}\text { Energy } E_{\mathrm{W}} \\
(\mathrm{mJ})\end{array}$ & $\begin{array}{l}\text { Power } P_{\mathrm{W}} \\
(\mathrm{MW})\end{array}$ & $\begin{array}{l}\text { Intensity } I_{\mathrm{W}} \\
\left.(\mathrm{GW} \mathrm{cm})^{-2}\right)\end{array}$ & $\begin{array}{l}\text { Fluence } \Phi_{\mathrm{W}} \\
\left(\mathrm{J} \mathrm{cm}^{-2}\right)\end{array}$ & $\begin{array}{l}\text { Photon flux } F_{\mathrm{ph}} \\
\left(\mathrm{ph} . \mathrm{cm}^{-2} \mathrm{~s}^{-1}\right)\end{array}$ & $\begin{array}{l}\text { Electric field } F_{\mathrm{E}} \\
\left.(\mathrm{MV} \mathrm{cm})^{-1}\right)\end{array}$ \\
\hline 9.621 & 2685 & 42.1 & 5.36 & 342 & $2.60 \times 10^{29}$ & 1.50 \\
9.621 & 2256 & 35.4 & 4.50 & 287 & $2.18 \times 10^{29}$ & 1.37 \\
9.621 & 1732 & 27.1 & 3.46 & 220 & $1.67 \times 10^{29}$ & 1.20 \\
9.621 & 1209 & 19.0 & 2.41 & 154 & $1.17 \times 10^{29}$ & 1.01 \\
9.621 & 503 & 7.88 & 1.00 & 64.0 & $4.86 \times 10^{28}$ & 0.65 \\
10.591 & 3161 & 49.5 & 6.31 & 402 & $3.36 \times 10^{29}$ & 1.63 \\
10.591 & 2145 & 33.6 & 4.28 & 273 & $2.28 \times 10^{29}$ & 1.34 \\
10.591 & 1481 & 23.2 & 2.96 & 189 & $1.58 \times 10^{29}$ & 1.11 \\
10.591 & 968 & 15.2 & 1.93 & 123 & $1.03 \times 10^{29}$ & 0.90 \\
10.591 & 624 & 9.78 & 1.25 & 79.5 & $6.64 \times 10^{28}$ & 0.72 \\
10.591 & 436 & 6.83 & 0.87 & 55.5 & $4.64 \times 10^{28}$ & 0.60 \\
\hline
\end{tabular}

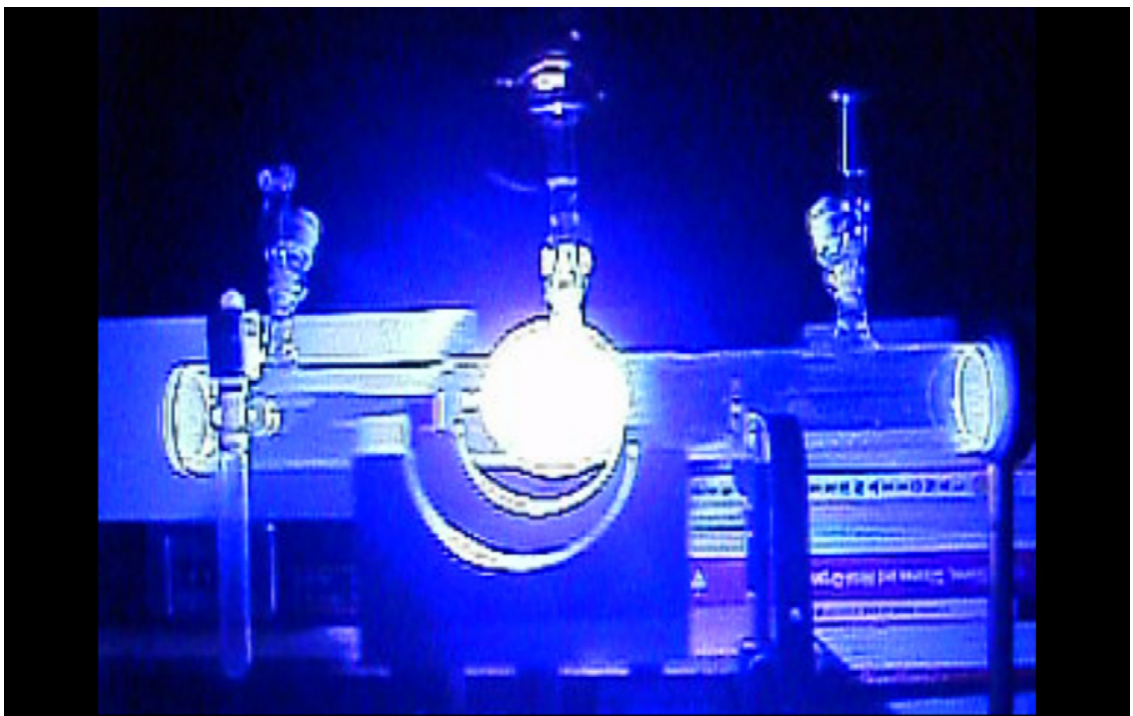

Figure 1. Laser-induced breakdown produced in oxygen at $49.0 \mathrm{kPa}$ by a TEA-CO $\mathrm{CO}_{2}$ laser pulse $(\lambda=10.591 \mu \mathrm{m}$ and power density of $1.93 \mathrm{GW} \mathrm{cm}^{-2}$ ). Laser beam direction is from right to left.

where $\pi r^{2}$ is the focal spot area $\left(\mathrm{cm}^{2}\right), \lambda$ is the laser wavelength, $h$ is the Planck constant, $c$ is the speed of light and $\varepsilon_{0}$ is the electric constant. The laser intensity, fluence, photon flux and electric field at the focal volume are inversely proportional to the focused spot size. For the present experiments, the LIB on oxygen is performed using a high-power IR $\mathrm{CO}_{2}$ pulsed laser at $\lambda=9.621$ and $\lambda=10.591 \mu \mathrm{m}, \tau_{\mathrm{FWHM}}=64 \mathrm{~ns}$, and the measured focusedspot area was $7.85 \times 10^{-3} \mathrm{~cm}^{2}$. For the different pulse laser energies measured in this work, the calculated laser peak power (equation (1)), intensity (equation (2)), fluence (equation (3)), photon flux (equation (4)) and electric field (equation (5)) are given in table 1.

\section{Results and discussion}

\subsection{Plasma visualization}

When a sample of oxygen is irradiated by $\mathrm{CO}_{2}$ laser lines of sufficient power, a visible bluish bright flash occurs. Figure 1 shows an example of an LIB plasma in oxygen induced by a single $\mathrm{CO}_{2}$ laser pulse. The cell was initially filled at room temperature with $\mathrm{O}_{2}$ at $49.0 \mathrm{kPa}$. The laser was focused on a point at the centre of the cell. A number of plasma lobes can be distinguished as well as diffuse, luminous cloud surrounding the central plasma core. The observations of the LIB geometry during the experiments indicate that the actual plasma region is not entirely spherical, but slightly elongated in the direction of the laser beam propagation.

\subsection{Identification of the chemical species in the laser-induced oxygen plasma}

To understand the detailed aspects of laser-beam interaction with the gas and recombination processes following the breakdown, time-resolved studies of the emission spectra from the plasma offer the most convenient approach. The emission spectrum has a continuum on which the emission lines are superimposed. This continuum extends over the whole range of wavelength and has been subtracted in our spectra.

Figures $2(a)-(f)$ display an overview of the lowresolution LIB emission spectrum (2320-9690 $\AA$ ) in oxygen at a pressure of $53.2 \mathrm{kPa}$, excited by the $10 \mathrm{P}(20)$ line of the $\mathrm{CO}_{2}$ laser, and assignment of the atomic lines of $\mathrm{O}, \mathrm{O}^{+}, \mathrm{O}^{2+}, \mathrm{N}$ and $\mathrm{N}^{+}$[29]. Strong atomic $\mathrm{O}$ lines dominate the spectrum but ionic $\mathrm{O}^{+}$lines (about 8 times weaker) and weak $\mathrm{O}^{2+}$ lines (about 


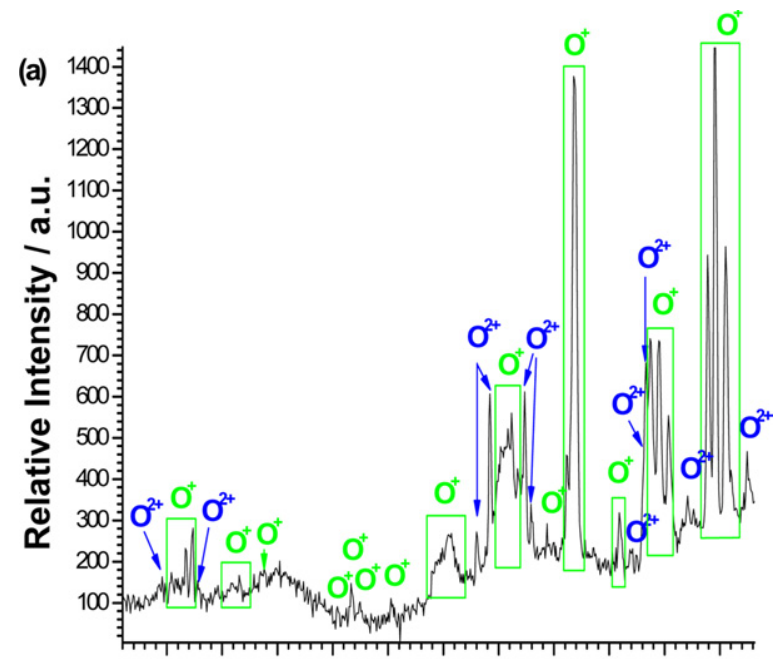

24002500260027002800290030003100320033003400

Air Wavelength/A

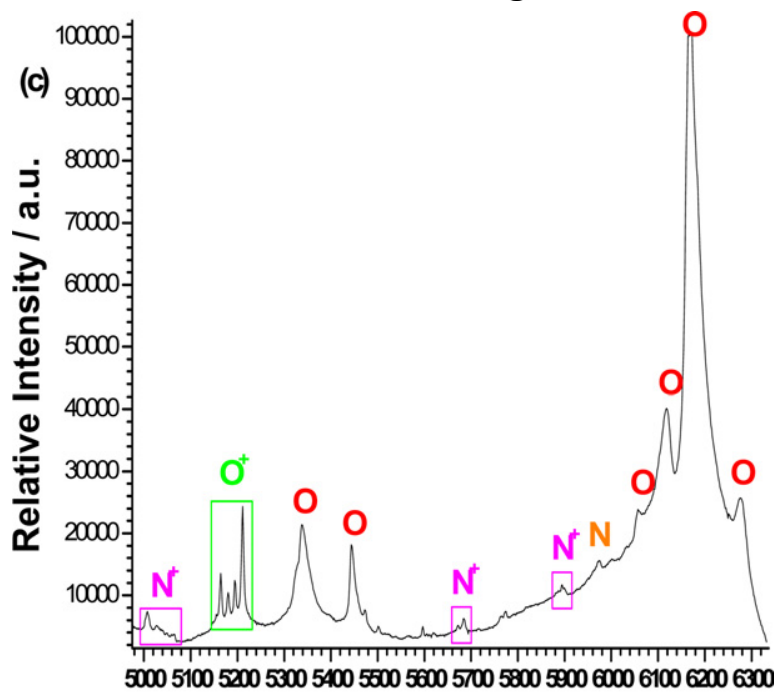

Air Wavelength/A

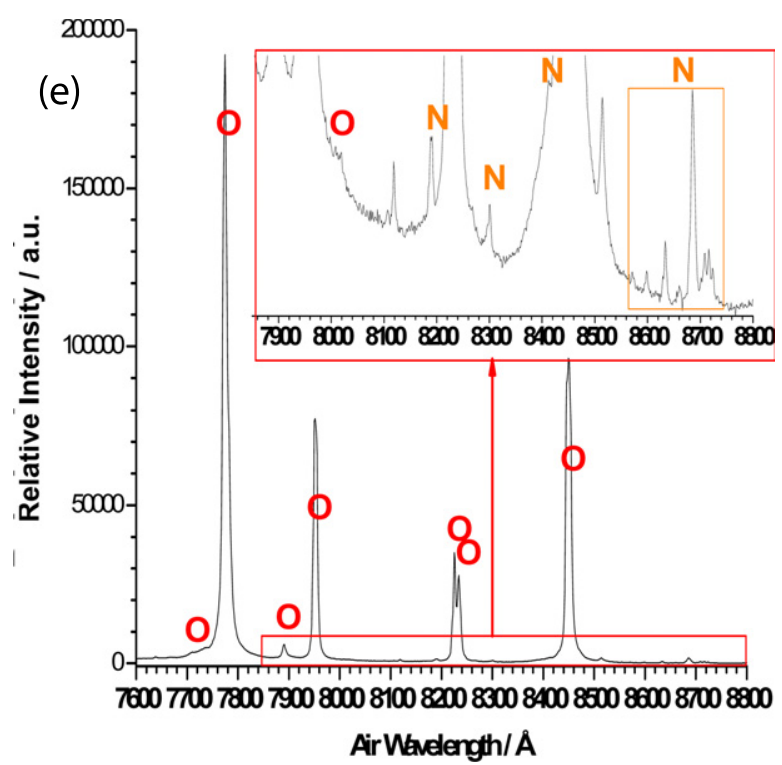

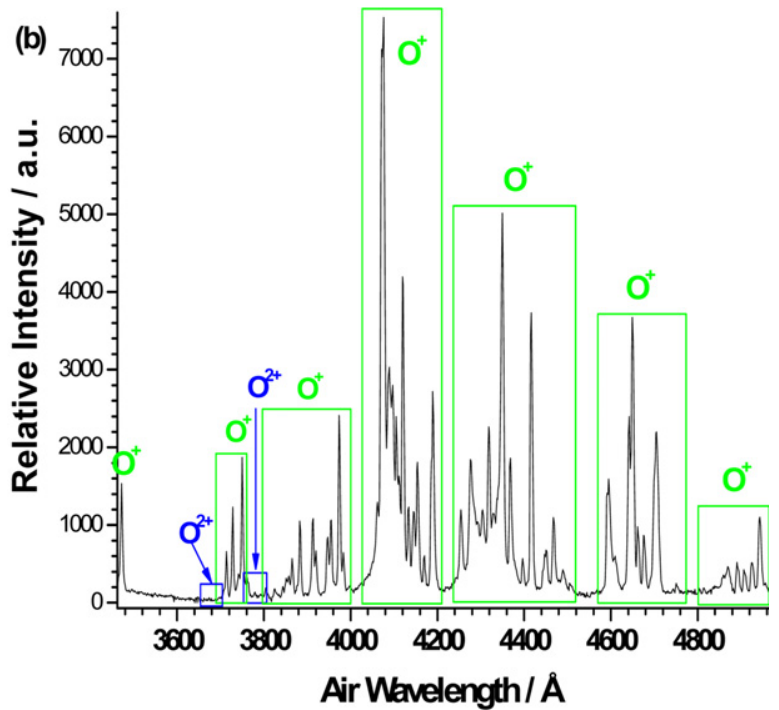
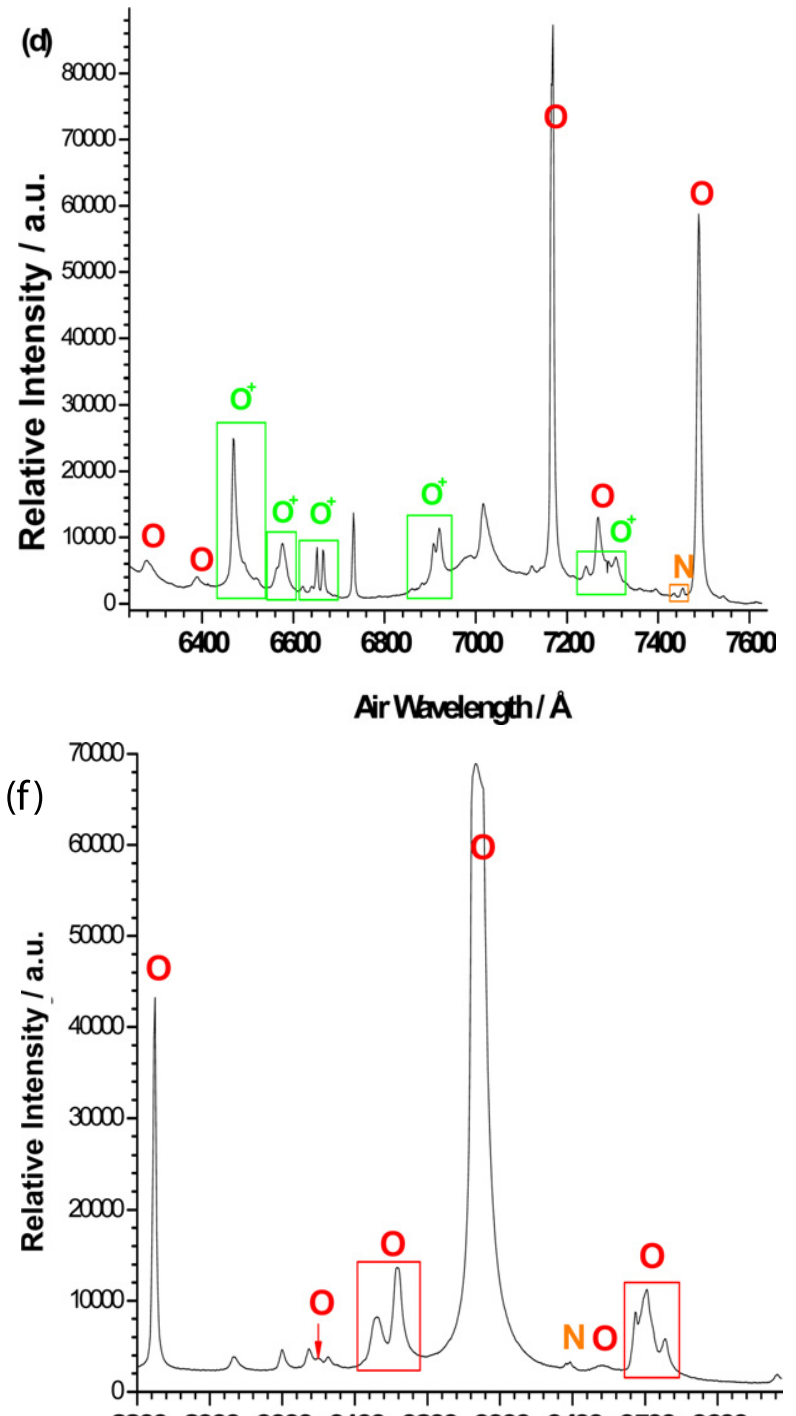

880089009000910092009300940095009600

Air Wavelength/ $\AA$

Figure 2. $(a)-(f)$ Low-resolution LIB emission spectrum observed in the 2320-9690 ̊ region in oxygen at a pressure of $53.2 \mathrm{kPa}$, excited by the $10 \mathrm{P}(20)$ line of the TEA- $\mathrm{CO}_{2}$ laser at $10.591 \mu \mathrm{m}$ and a power density of $0.87 \mathrm{GW} \mathrm{cm}^{-2}$, and assignment of the atomic lines of $\mathrm{O}, \mathrm{O}^{+}$, $\mathrm{O}^{2+}, \mathrm{N}$ and $\mathrm{N}^{+}$. 
150 times weaker) are also present. Atomic and ionic nitrogen lines were present, as well as the first negative band system $330-400 \mathrm{~nm}$ corresponding to the transition $B^{2} \Sigma_{\mathrm{u}}^{+}-X^{2} \Sigma_{\mathrm{g}}^{+}$in $\mathrm{N}_{2}^{+}$. In the acquisition of the spectrum of figures $2(d)-(f)$ a cut-off filter was used to suppress the second order intense UV oxygen atomic lines. This cut-off filter produces a decrease in the intensity with regard to the spectra of figures $2(a)-(c)$. In order to get more insight into LIB of oxygen and to obtain an unambiguous assignment of the emission lines, we have scanned the corresponding wavelength regions with a higher resolution $(\sim 0.10 \AA$ in first-order $)$, which was sufficient to distinguish clearly between nearly all observed lines. The spectra have been obtained with fifty successive exposures on the ICCD camera in the spectral region 1900-7500. As examples, figures $3(a)-(f)$ show several spectra recorded in the LIB experiment. These LIB emission spectra were recorded under the following experimental conditions: oxygen pressure of $48.8 \mathrm{kPa}$, excitation line $10 \mathrm{P}(20)$ at $10.591 \mu \mathrm{m}$ and $\mathrm{CO}_{2}$ laser power density $4.28 \mathrm{GW} \mathrm{cm}^{-2}$. Figures $3(a)-(f)$ display some details of the large features found in figures $2(a)$ $(f)$. No new features were observed in these high-resolution spectra. In these figures, multiplet transitions between different $J$ levels for $\mathrm{O}^{+}$and $\mathrm{O}^{2+}$ are observed. In some cases these multiplet structures are not completely resolved due to Stark broadening of atomic lines. The relative intensities of the observed emission lines reasonably agree with the tabulated values in the NIST Atomic Spectral Database [29]. The spectral features clearly show the complexity of the relaxation process and bring out the possibility of cascading processes.

\subsection{Plasma excitation temperature measurement}

We have calculated the excitation temperature $T_{\text {exc }}$ according to the Boltzmann equation under the assumption of local thermodynamic equilibrium (LTE). The conditions satisfying this assumption of LTE require that the radiative depopulation rates are negligible compared with the collisional depopulation rates. This essentially implies that an excited state must have a higher probability of de-excitation by collision than by spontaneous emission. Generally LTE is established among all the species in the plasma beyond $\sim 1 \mu$ s delay time from the plasma start.

The excitation temperature was calculated from the relative intensities of several $\mathrm{O}^{+}$(3270-3310 $\AA$ spectral region) and $\mathrm{O}^{2+}(2900-3350 \AA$ spectral region) atomic lines and the slope of the Boltzmann plot $\ln \left[I_{k i} \cdot \lambda_{k i} / g_{k} \cdot A_{k i}\right]$ versus $E_{k} / k_{\mathrm{B}}$ :

$$
\ln \left[\frac{I_{k i} \cdot \lambda_{k i}}{g_{k} \cdot A_{k i}}\right]=C-\frac{E_{k}}{k_{\mathrm{B}} \cdot T_{\mathrm{exc}}},
$$

where $I_{k i}$ is the emissivity in $\mathrm{W} \mathrm{m}^{-3} \mathrm{sr}^{-1}$ of the emitted $k \rightarrow i$ spectral line, $\lambda_{k i}$ is the wavelength, $g_{k}=2 J_{k}+1$ is the statistical weight, $A_{k i}$ is the Einstein transition probability of spontaneous emission, $E_{k} / k_{\mathrm{B}}$ is the normalized energy of the upper electronic level and $C=\ln \left(h c N_{k} / 4 \pi Q(T)\right)(Q(T)$ is the partition function). The values of $\lambda_{k i}, g_{k}, A_{k i}$ and $E_{k}$ for $\mathrm{O}^{+}$and $\mathrm{O}^{2+}$ selected atomic lines were obtained from the NIST Atomic Spectral Database [29]. The excitation temperatures were determined under the following experimental conditions: oxygen pressure of $48.8 \mathrm{kPa}$, excitation line $10 \mathrm{P}(20)$ at $10.591 \mu \mathrm{m}$ and $\mathrm{CO}_{2}$ laser power density $4.28 \mathrm{GW} \mathrm{cm}^{-2}$. The obtained excitation temperatures, in the case of $\mathrm{O}^{+}$and $\mathrm{O}^{2+}$, were $23000 \pm 3000 \mathrm{~K}$ and $31500 \pm 1600 \mathrm{~K}$, respectively (figure 4). This behaviour can be due to the different quenching rate coefficients between each species. Also this fact may be interpreted to result from the different emissivity distributions of single ionized and double ionized oxygen lines. The emissivity of the double ionized lines is produced, on average, near the laser-induced plasma inner region with higher temperature. In contrast, the emissivity of the single ionized lines comes, on average, from the low-temperature region close to the plasma front, where the double ionized atom density is lower. The intensity measurements correspond to the integration of the local emissivity values along the line of sight, integrated in turn in the perpendicular directions. As a consequence, the single ionized oxygen Boltzmann plot provides a temperature value which is a certain average of the low-temperature values in the plasma $(23000 \mathrm{~K})$, whereas the temperature obtained from the double ionized Boltzmann plot $(31500 \mathrm{~K})$ averages the values existing in the hightemperature region. Keeping in mind these results, the temperature obtained from the relative intensity of $\mathrm{O}^{+}$atomic lines $(23000 \mathrm{~K})$ was chosen as the first approximation for the excitation temperature. Real plasmas provide, even in the worst cases, just an approximation to LTE. However, their spatial inhomogeneity is very large as concerns the temperature and number density distributions of the different species. Consequently, the LTE occurs only within a very small volume of the plasma.

\subsection{Ionization degree of the plasma}

When dielectric breakdown is produced in oxygen under high intensity laser radiation, some molecules can obtain an energy that exceeds the binding energy. Also some of their electrons become so energetic that the atoms and molecules ionize. Taking into account our experimental spectral observations, at these high temperatures oxygen becomes a mixture mainly of primary $\mathrm{O}_{2}, \mathrm{O}, \mathrm{O}^{+}, \mathrm{O}^{2+}$ and electrons. The transition between a gas and plasma is essentially a chemical equilibrium which shifts from the gas to the plasma side with increasing temperature. Let us consider the first two different ionization equilibria of oxygen:

$$
\begin{gathered}
\mathrm{O}\left(2 \mathrm{~s}^{2} 2 \mathrm{p}^{4}{ }^{3} \mathrm{P}_{2}\right) \leftrightarrow \mathrm{O}^{+}\left(2 \mathrm{~s}^{2} 4 \mathrm{p}^{3}{ }^{4} \mathrm{~S}_{3 / 2}^{\mathrm{o}}\right)+\mathrm{e}+\mathrm{I}_{\mathrm{P}}(\mathrm{O}-\mathrm{I}), \\
\mathrm{O}^{+}\left(2 \mathrm{~s}^{2} 2 \mathrm{p}^{3}{ }^{4} \mathrm{~S}_{3 / 2}^{\mathrm{o}}\right) \leftrightarrow \mathrm{O}^{2+}\left(2 \mathrm{~s}^{2} 2 \mathrm{p}^{2}{ }^{3} \mathrm{P}_{0}\right)+\mathrm{e}+I_{\mathrm{P}}(\mathrm{O}-\mathrm{II}),
\end{gathered}
$$

where the first two ionization potentials (IPs) for oxygen are $I_{\mathrm{P}}(\mathrm{O}-\mathrm{I})=13.618 \mathrm{eV}$ and $I_{\mathrm{P}}(\mathrm{O}-\mathrm{II})=35.121 \mathrm{eV}$ [30]. For each ionization equilibrium, the LTE between ionization and recombination reactions at temperature $T$ is described by the Saha equation

$$
\frac{n_{\mathrm{e}} \cdot N_{\mathrm{i}}}{N_{0}}=\frac{g_{\mathrm{e}} \cdot g_{\mathrm{i}}}{g_{0}} \frac{\left(2 \pi m_{\mathrm{e}} k_{\mathrm{B}} T\right)^{3 / 2}}{h^{3}} \mathrm{e}^{-I_{\mathrm{P}} / k_{\mathrm{B}} T},
$$

where $n_{\mathrm{e}}=N_{\mathrm{i}}$ are the electron and ion densities in different ionization equilibria in the second member of ionization 


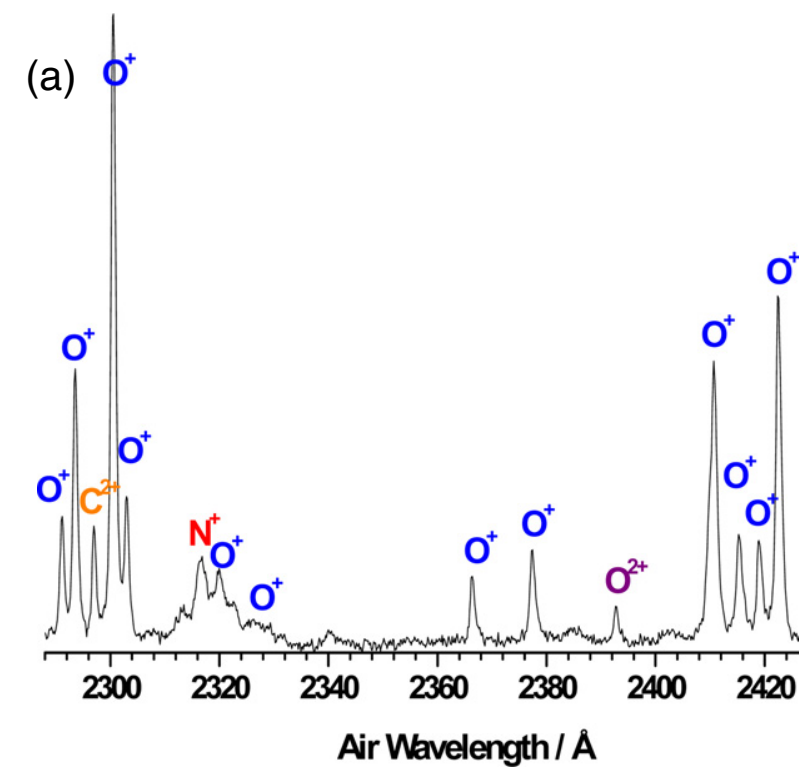

(b)

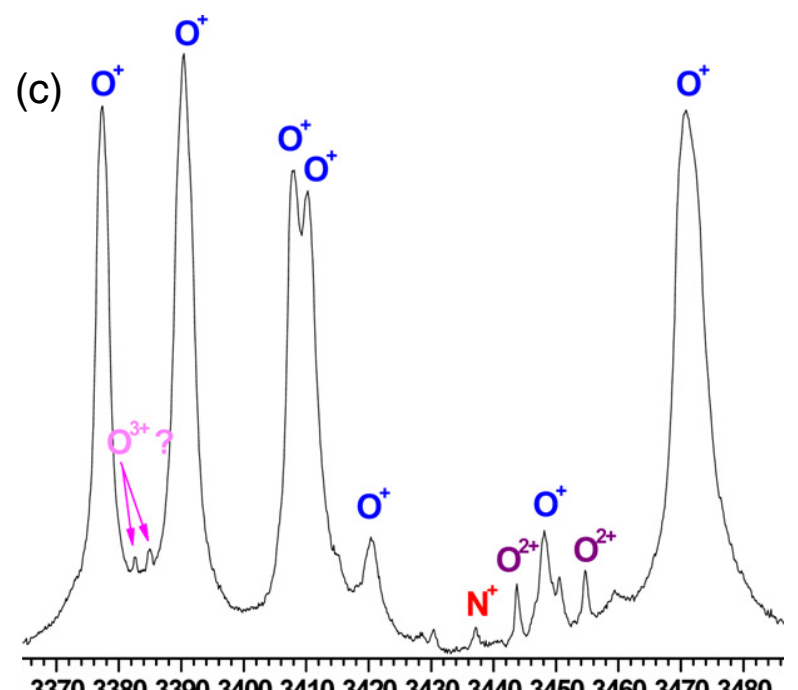

337033803390340034103420343034403450346034703480

Air Wavelength / A

$\mathrm{O}^{+}$

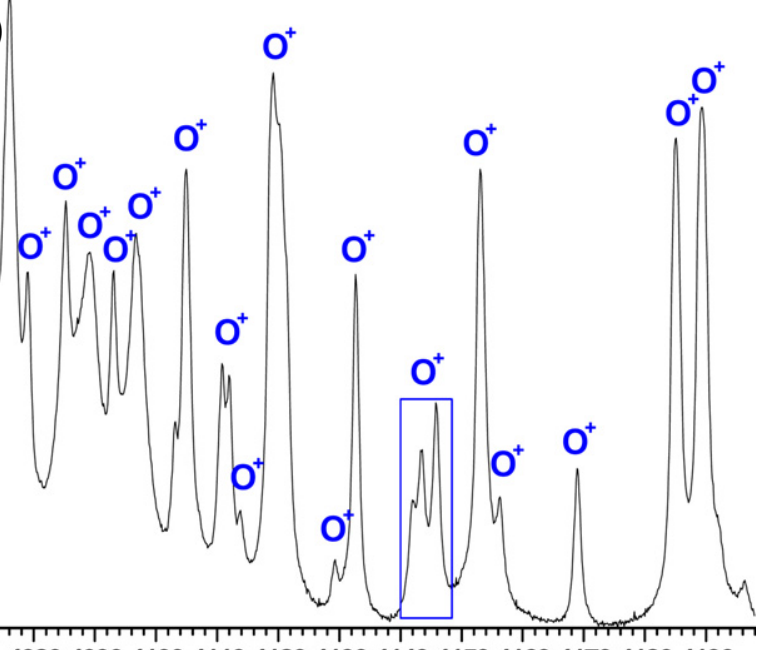

(e)

408040904100411041204130414041504160417041804190

\section{Air Wavelength / $\AA$}

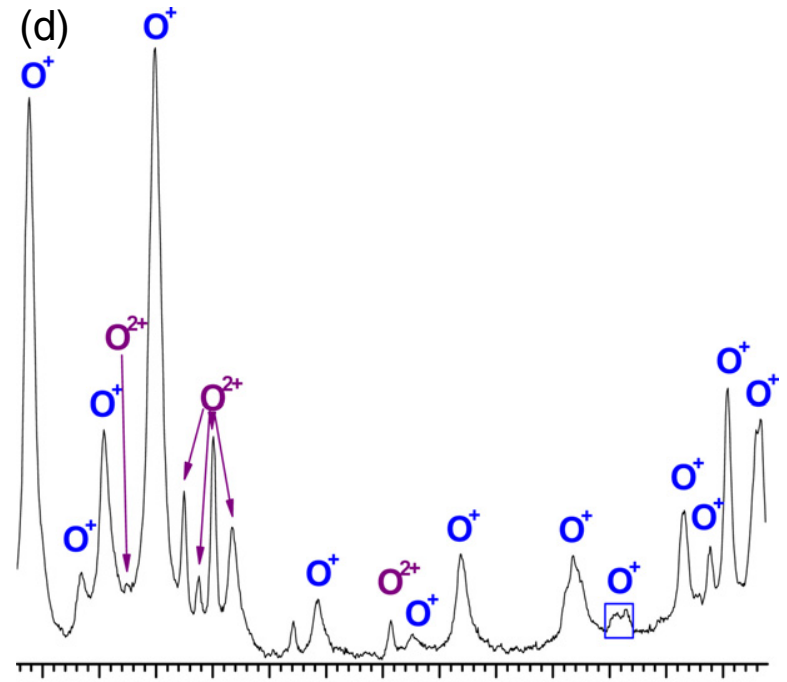

3730374037503760377037803790380038103820383038403850

\section{Air Wavelength / A}

(f)

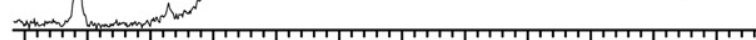

323032403250326032703280329033003310332033303340

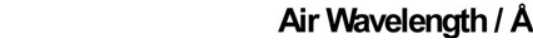

(d)

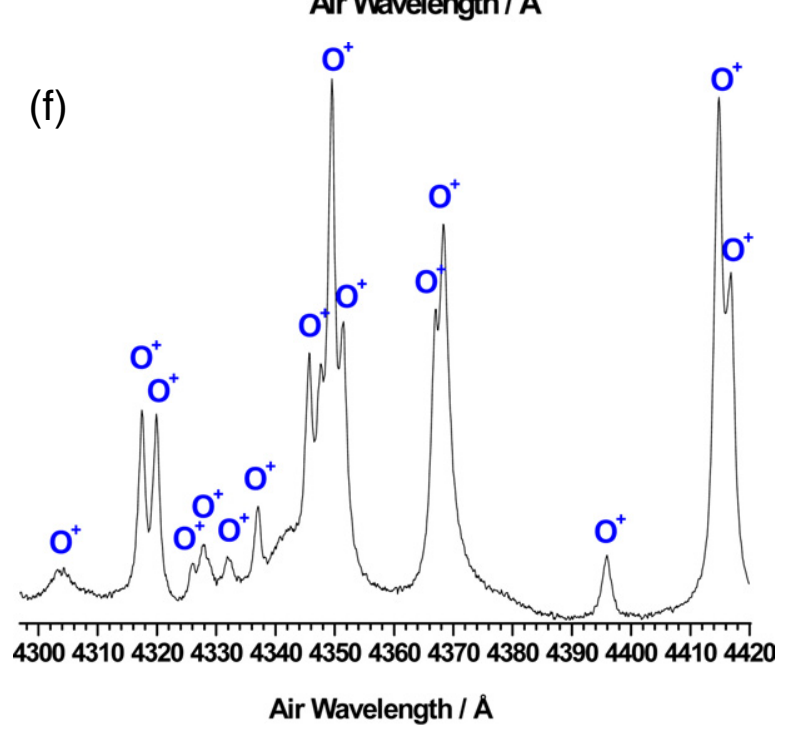

Figure 3. $(a)-(f)$ High-resolution LIB emission spectrum observed in several spectral regions in oxygen at a pressure of $48.8 \mathrm{kPa}$, excited by the $\mathrm{CO}_{2}$ laser at $10 \mathrm{P}(20)$ line $(10.591 \mu \mathrm{m})$ with a power density of $4.28 \mathrm{GW} \mathrm{cm}^{-2}$, and assignment of some atomic lines of $\mathrm{O}^{+}, \mathrm{O}^{2+}$, and $\mathrm{N}^{+}$. 

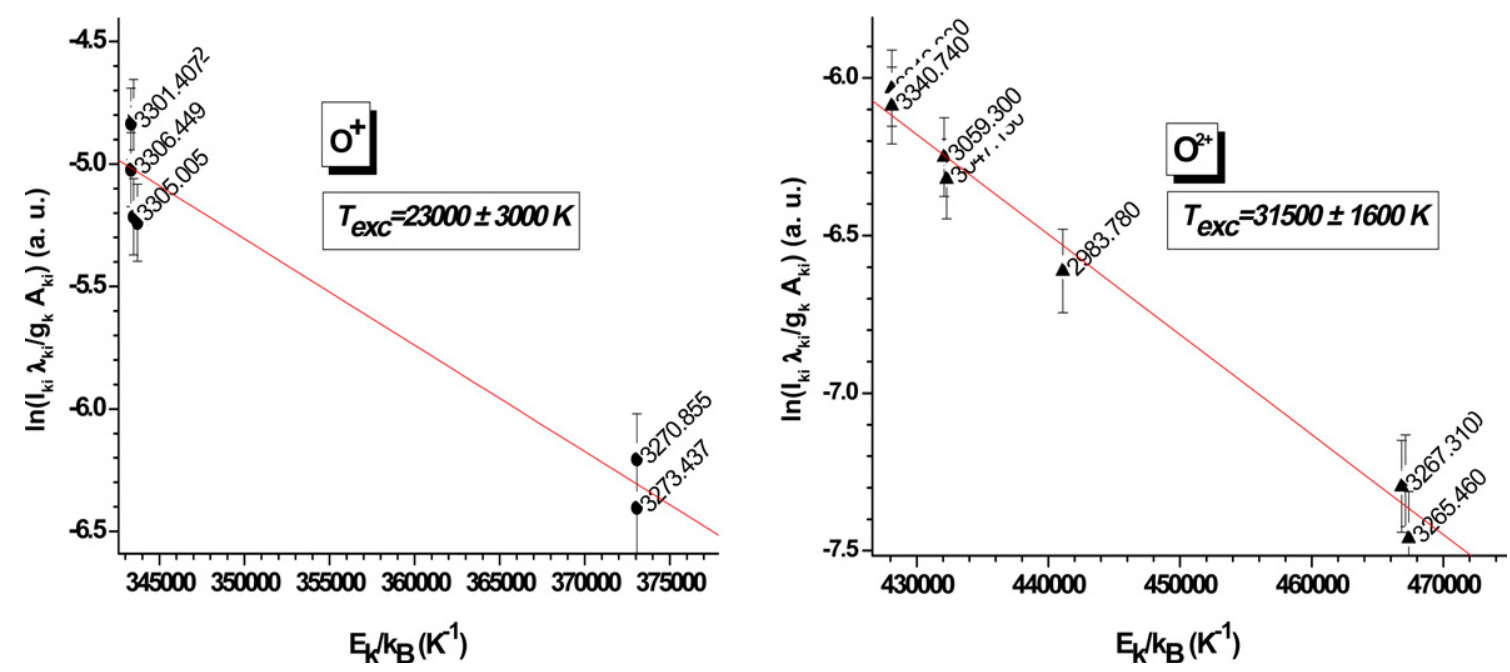

Figure 4. Linear Boltzmann plots for several $\mathrm{O}^{+}$and $\mathrm{O}^{2+}$ transition lines used to calculate plasma temperature, $T_{\text {exc }}$. Plots also show a linear fit to the data.

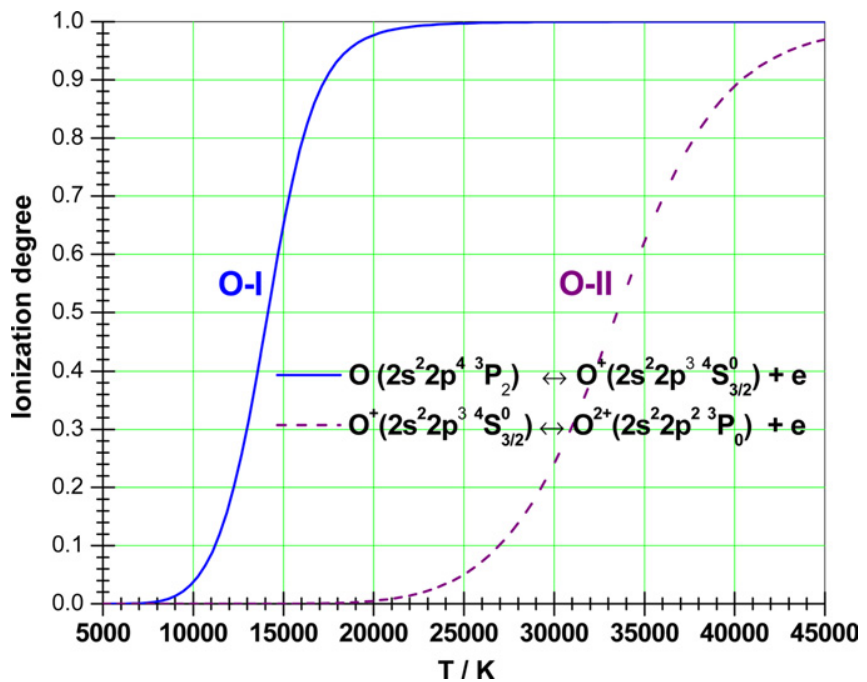

Figure 5. Temperature dependence of the ionization degree $N_{\mathrm{i}} /\left(N_{0}+N_{\mathrm{i}}\right)$ of atomic oxygen $\mathrm{O}$ and oxygen singly ionized $\mathrm{O}^{+}$at a constant pressure of $53.2 \mathrm{kPa}$.

equilibria, $N_{0}$ the density of the oxygen or ions in the first member of ionization equilibria, $h$ is Planck's constant, $k_{\mathrm{B}}$ is Boltzmann's constant, $m_{\mathrm{e}}$ the electron mass and $g_{\mathrm{e}}, g_{\mathrm{i}}$ and $g_{0}$ the statistical weights of the electrons $\left(g_{\mathrm{e}}=2\right), \mathrm{O}^{+}$ions $\left(g_{\mathrm{i}}=4\right), \mathrm{O}^{2+}$ ions $\left(g_{\mathrm{i}}=1\right)$ and $\mathrm{O}$ neutrals $\left(g_{0}=5\right)$. The Saha equation reads

$$
\frac{n_{\mathrm{e}} \cdot N_{\mathrm{i}}}{N_{0}}=C T^{3 / 2} \mathrm{e}^{-I_{\mathrm{P}} / k_{\mathrm{B}} T}
$$

where $C=3.864 \times 10^{21} \mathrm{~K}^{-2 / 3} \mathrm{~m}^{-3}$ and $1.207 \times$ $10^{21} \mathrm{~K}^{-2 / 3} \mathrm{~m}^{-3}$ for the first two ionization equilibria of oxygen, respectively, and $T$ is in $\mathrm{K}$. Figure 5 shows the ionization degree $N_{\mathrm{i}} /\left(N_{0}+N_{\mathrm{i}}\right)$ of $\mathrm{O}$ and $\mathrm{O}^{+}$, plotted as a function of the gas temperature $T$ at a constant total pressure $P=$ $\left(N_{0}+n_{\mathrm{e}}+N_{\mathrm{i}}\right) k_{\mathrm{B}} T$ of $53.2 \mathrm{kPa}$. The graph shows that oxygen is already fully ionized at thermal energies well below the first ionization energy of $13.618 \mathrm{eV}$ (equivalent to $158000 \mathrm{~K}$ ). If we consider a temperature of $23000 \mathrm{~K}$, the ionization degrees of $\mathrm{O}$ and $\mathrm{O}^{+}$obtained by means of the Saha equation (8) are 0.994 and 0.022 , respectively. For $T=31500 \mathrm{~K}$, the ionization degrees of $\mathrm{O}$ and $\mathrm{O}^{+}$obtained by means of the Saha equation (8) are 0.999 and 0.34 , respectively. These so high values of the ionization degrees justify the observed emission spectra.

\subsection{Electron number density}

The evolution of the laser plasma can be divided into several transient phases. The initial plasma $(\sim 0-100 \mathrm{~ns})$ is characterized by high electron and ion densities $\left(10^{17}\right.$ $10^{20} \mathrm{~cm}^{-3}$ ) and temperatures around $40000 \mathrm{~K}$. Of course, the density gradient of the plasma is the highest in the earliest time of plume expansion. The emission spectrum for the early stage of the plasma is characterized by a continuum background emission mainly due to bremsstrahlung and recombination processes of electrons with ion in the plasma. Structured emission spectra from atomic and ionized species can be found after $\sim 300 \mathrm{~ns}$ delay. The observed spectral lines are always broadened, partly due to the finite resolution of the spectrometer and partly due to intrinsic physical causes. The principal physical causes of spectral line broadening are the Doppler, resonance pressure and Stark broadening. The Doppler broadening is due to the thermal motion of the emitting atoms or ions. For a Maxwellian velocity distribution the line shape is Gaussian, and the FWHM may be estimated as (in $\AA$ ) $\Delta \lambda_{\mathrm{FWHM}}^{\mathrm{D}}=7.16 \times 10^{-7} \cdot \lambda \cdot \sqrt{T / M}$, with $\lambda$ the wavelength in $\AA$, $T$ the temperature of the emitters in $\mathrm{K}$ and $M$ the atomic mass in amu. The Doppler line widths for some lines of $\mathrm{O}^{+}$for different temperatures are shown in figure 6 . In our experiments, for $\mathrm{O}^{+}$ lines, the Doppler line widths are $0.07-0.12 \AA$ at $23000 \mathrm{~K}$.

Owing to the high electron densities in LIBS experiments, the emission lines are mainly broadened by the Stark effect. Both ions and electrons produce Stark broadening, but electrons are responsible for the major part because of their higher relative velocities. Stark broadening produces Lorentzian line shapes, except at the line centre, where electrostatic interactions with ions cause a dip. The FWHM 


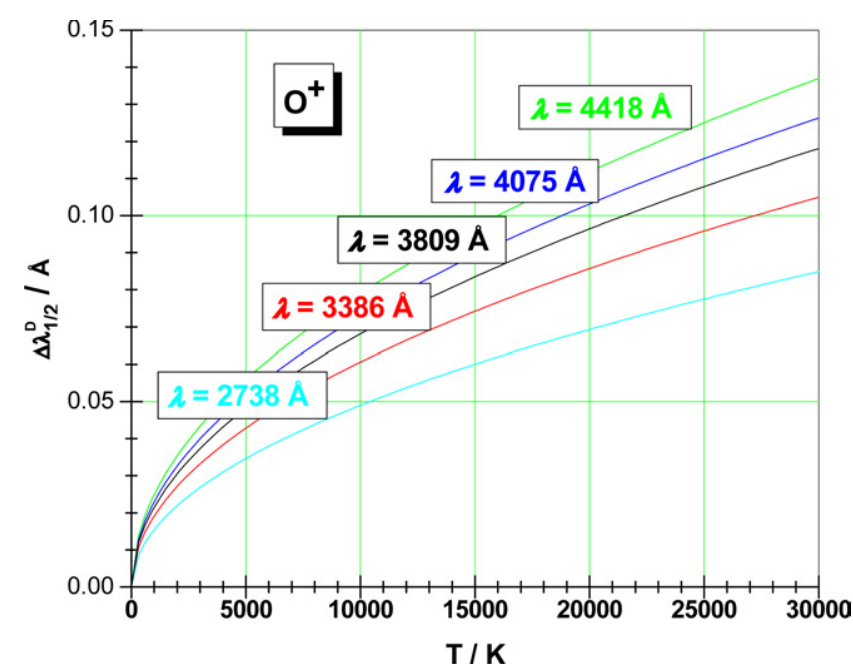

Figure 6. Doppler line widths for some lines of $\mathrm{O}^{+}$.

of the Stark broadened lines is related to the electron number density $n_{\mathrm{e}}\left(\mathrm{cm}^{-3}\right)$ by [31]

$$
\begin{gathered}
\Delta \lambda_{\mathrm{FWHM}}^{S}=2 W\left(\frac{n_{\mathrm{e}}}{10^{16}}\right)+3.5 A\left(\frac{n_{\mathrm{e}}}{10^{16}}\right)^{1 / 4} \\
\quad \times\left(1-B N_{\mathrm{D}}^{-1 / 3}\right) W\left(\frac{n_{\mathrm{e}}}{10^{16}}\right),
\end{gathered}
$$

where $W$ is the electron impact parameter or half-width, $A$ is the ion impact parameter both in $\AA, B$ is a coefficient equal to 1.2 or 0.75 for ionic or neutral lines, respectively, and $N_{\mathrm{D}}$ is the number of particles in the Debye sphere:

$$
N_{\mathrm{D}}=1.72 \times 10^{9} \frac{T^{3 / 2}}{n_{\mathrm{e}}^{1 / 2}} .
$$

The electron and ion impact parameters are functions of temperature. The first term on the right side of equation (9) refers to the broadening due to the electron contribution, whereas the second one is the ion broadening. Since for LIB conditions Stark broadening is predominantly by electron impact, the ion correction factor can safely be neglected, and equation (9) becomes

$$
\Delta \lambda_{\mathrm{FWHM}}^{S}=2 W\left(\frac{n_{\mathrm{e}}}{10^{16}}\right) .
$$

The choice of plasma emission for $n_{\mathrm{e}}$ measurements is made to ensure that the $\mathrm{O}^{+}$spectral lines are sensitive enough to Stark effect and do not suffer from interference by other species. In our case, the estimation of electron density $n_{\mathrm{e}}$ has been carried out by measuring the broadening of the spectral profiles of isolated lines of $\mathrm{O}^{+}(2738,3386$, 3809, 4075 and $4418 \AA$ ) from the high-resolution spectra. The electron impact parameters for the different $\mathrm{O}^{+}$lines were approximated to a first-order exponential decay. The electron number densities of the laser-induced plasma were determined from the high-resolution emission spectra in oxygen at a pressure of $48.8 \mathrm{kPa}$, excited by the $\mathrm{CO}_{2}$ laser at $10.591 \mu \mathrm{m}$ with a power density of $4.28 \mathrm{GW} \mathrm{cm}^{-2}$. A Lorentz function was used to fit the spectra. In order to extract the Stark broadening from the total experimentally measured line broadening, we have to previously deconvolute the different effects that contribute to the broadening of the spectral line: the instrumental, Doppler and Stark broadenings. Values of the electron impact half-width $W$ were taken from the extensive tables given by Griem [32]. Electron densities in the range $(3.5-16.5) \times 10^{16} \mathrm{~cm}^{-3}$, with an estimated uncertainty of $10 \%$, were determined from the Stark broadening data of several singly ionized oxygen lines. The validity criteria for LTE and, consequently, criteria for the application of the different spectroscopic methods are extensively discussed in the literature $[33,34]$. For the LTE, the excited states have to be populated through collisions. Using McWhirter's criterion [34] to check the condition for the validity of the LTE, we find that the electron number density satisfies

$$
n_{\mathrm{e}} \geqslant 1.6 \times 10^{12} T^{1 / 2}(\Delta E)^{3} .
$$

Here, $n_{\mathrm{e}}\left(\mathrm{cm}^{-3}\right)$ is the electron density, $T(\mathrm{~K})$ is the plasma temperature and $\Delta E(\mathrm{eV})$ is the difference in the energies between the upper and lower states of all the $\mathrm{O}^{+}$investigated transitions. At the evaluated temperature of $23000 \pm 3000 \mathrm{~K}$, equation (12) yields $n_{\mathrm{e}} \approx(0.54-2.3) \times 10^{16} \mathrm{~cm}^{-3}$. These electron densities are lower than the deduced values from the Stark broadening $(3.5-16.5) \times 10^{16} \mathrm{~cm}^{-3}$, approximately one order of magnitude. Based on these calculations, the validity of the LTE assumption is supported.

\subsection{Effect of laser wavelength and irradiance}

The interactions between the incoming laser radiation and the gas sample depend upon numerous variables related to the laser and the gas. These variables include laser wavelength, energy, spatial and temporal profile of the laser beam and the thermal properties of the sample. The incident beam is partially reflected and partially absorbed by the bulk to a degree that depends on the nature of the gas and the temperature it reaches under laser irradiation.

LIBS spectra obtained by laser irradiation at different wavelengths are compared in figure 7 . These high-resolution LIB emission spectra in oxygen were obtained to a pressure of $48.8 \mathrm{kPa}$, excited by two TEA- $\mathrm{CO}_{2}$ laser wavelengths at $10.591 \mu \mathrm{m}\left(I_{\mathrm{W}}=6.31 \mathrm{GW} \mathrm{cm}^{-2}\right)$ and $9.621 \mu \mathrm{m}\left(I_{\mathrm{W}}=\right.$ $\left.5.36 \mathrm{GW} \mathrm{cm}^{-2}\right)$. The spectral range was chosen in order to detect both single and double ionized oxygen species. Also this spectral region has been selected to show differences in signal intensity and background emission in detail. The first remark that we should make is that the background continuum emission after the same optimization performed on the data acquisition window is much stronger for the spectrum produced by the $10.591 \mu \mathrm{m}$ laser line. This is due to the higher laser power density $\left(I_{\mathrm{W}}=6.31 \mathrm{GW} \mathrm{cm}^{-2}\right)$ and the higher absorption in the plasma caused by the inverse bremsstrahlung, whose cross-section is proportional to $\lambda^{3}[1-$ $\left.\exp \left(-h c / \lambda k_{\mathrm{B}} T\right)\right]^{-1}$ or approximately $\lambda^{2}$ ( $T$ is the electron temperature during the electron avalanche or cascade growth of ionization and $\lambda$ is the laser excitation wavelength). The spectral lines of $\mathrm{O}^{+}$and $\mathrm{O}^{2+}(2983.78,3017.63,3023.45$, $3043.02,3047.13,3059.30 \AA$ ) were clearly observed. It is clear that the ionic spectral lines for both $\mathrm{O}^{+}$and $\mathrm{O}^{2+}$ were 


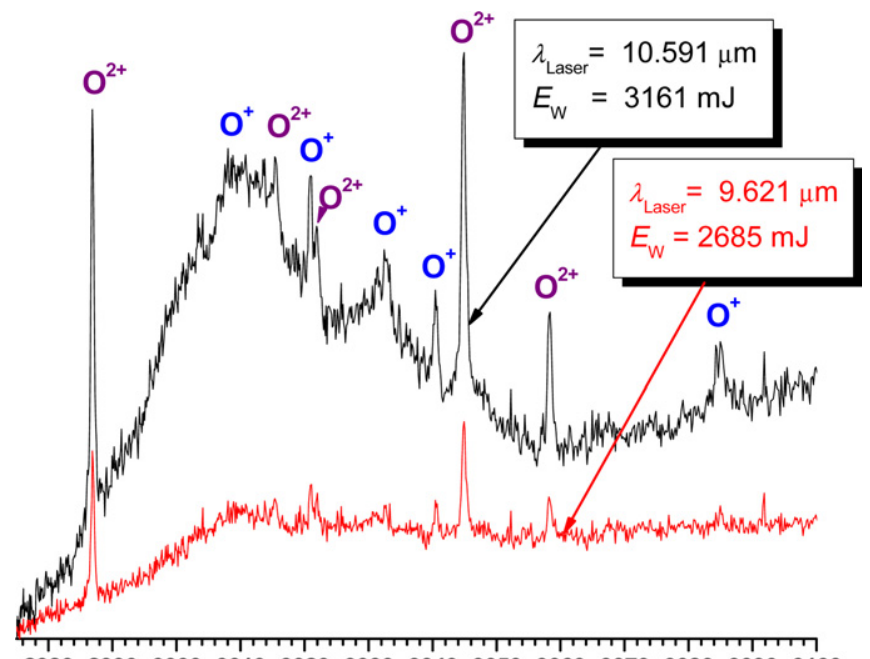

$\begin{array}{lllllllllllll}2980 & 2990 & 3000 & 3010 & 3020 & 3030 & 3040 & 3050 & 3060 & 3070 & 3080 & 3090 & 3100\end{array}$

Air Wavelength / A

Figure 7. High-resolution LIB emission spectra in oxygen at a pressure of $48.8 \mathrm{kPa}$, excited by two TEA-CO $\mathrm{CO}_{2}$ laser wavelengths at $10.591 \mu \mathrm{m}\left(E_{\mathrm{W}}=3161 \mathrm{~mJ}\right)$ and $9.621 \mu \mathrm{m}\left(E_{\mathrm{W}}=2685 \mathrm{~mJ}\right)$.

enhanced by a factor of 4 when the LIBS is induced by the TEA-CO $\mathrm{CO}_{2}$ laser at $10.591 \mu \mathrm{m}$. Besides, the irradiation at this wavelength favours the formation of doubly ionized species ( $\sim 30 \%)$, as it is obtained from the ratio of the intensities of the $\mathrm{O}^{2+}$ and $\mathrm{O}^{+}$lines. Moreover, plasma electron densities were determined from Stark broadening of the $\mathrm{O}^{2+}$ double ionized line at $2983.78 \AA$. The measured electron densities for the LIB emission spectra in oxygen were $(3.5 \pm 0.2) \times 10^{16} \mathrm{~cm}^{-3}$ and $(3.2 \pm 0.2) \times 10^{16} \mathrm{~cm}^{-3}$ for excitation at $10.591 \mu \mathrm{m}$ and $9.621 \mu \mathrm{m}$, respectively. Values of the electron impact halfwidth $W$ for $\mathrm{O}^{2+}$ were taken from the reported values given by Sreckovic et al [35]. It is noted that the emission intensity for $\mathrm{O}^{+}$and $\mathrm{O}^{2+}$ shows a different picture from the electron density possibly due to the effect of the laser wavelength. To see the effect of laser irradiance the measurements were also carried out at different laser fluences. Optical emission spectra of the oxygen plasma plume at a pressure of $48.8 \mathrm{kPa}$ as a function of the laser intensity are shown in figures $8(a)$ and $(b)$. These spectra were recorded after the incidence of only one pulse of the TEA- $\mathrm{CO}_{2}$ laser. The data were measured at a delay of $20 \mathrm{~ms}$. An increase in atomic/ionic emission intensity and in the doubly ionized $\mathrm{O}^{2+}$ formation with respect to $\mathrm{O}^{+}$with increasing laser irradiance was observed. Also the background increases with the laser power. At higher laser power densities $\left(6.31-4.28 \mathrm{GW} \mathrm{cm}^{-2}\right)$, the spectral lines are more broadened than at lower power densities as a result of the high pressure associated with the plasma. It is assumed that at a higher laser fluence the LIB plasma is more energetic and more ionized.

\subsection{Effect of oxygen pressure on the plasma}

The emission characteristics of the laser-induced plasma are influenced by the composition of the gas atmosphere. The pressure of the gas is one of the controlling parameters of the plasma characteristics, as well as the factors related to the laser energy absorption. Also the presence of air gas (vacuum conditions) during the LIB process has consequences on the expansion dynamics. An interesting observation was the effect of the oxygen pressure. Nanosecond TEA $\mathrm{CO}_{2}$ laser produced plasma emission has been characterized as a function of oxygen pressure. Experiments were performed in the pressure interval from 8 to $50 \mathrm{kPa}$ and at a pulse energy of $3161 \mathrm{~mJ}$. Figure 9 shows LIB emission spectra at various oxygen pressures, excited by the $10.591 \mu \mathrm{m}$ line at a power density of $6.31 \mathrm{GW} \mathrm{cm}^{-2}$. As can be seen in figure 9, the intensities of different spectral lines of $\mathrm{O}^{+}$and $\mathrm{O}^{2+}$ increase with decreasing pressure, reach a maximum at about $12.5 \mathrm{kPa}$ and then decrease for lower pressures. Characteristic emission lines from $\mathrm{O}^{+}$and $\mathrm{O}^{2+}$ elements exhibited significant enhancement in the signal intensity at a few $\mathrm{kPa}$ oxygen pressure as compared with high pressures below atmospheric pressure. However, the ratio of the intensities among the $\mathrm{O}^{2+}$ and $\mathrm{O}^{+}$lines remains the same at all pressures. The measurements indicate enhancement due to a longer lifetime of the plasma expanding to a larger size at lower oxygen pressures. Further reduction in oxygen pressure down to $\sim 12.5 \mathrm{kPa}$ resulted in a decrease in signal intensity, as a result of a reduction in collisional excitation of the emission lines which occurs when the plasma plume expands into the oxygen atmosphere.

\subsection{Optical breakdown threshold intensities for $\mathrm{O}_{2}$}

The strong atomic and ionic lines observed in LIBS spectra of oxygen indicate the high degree of excitation/ionization and the high rate of the $\mathrm{O}_{2}$ dissociation process achieved in the plasma. When a high-power laser beam of intensity $I_{\mathrm{W}}$ interacts with a gas, electrons involving the formation of laserinduced plasma can be generated through two main processes: multiphoton ionization (MPI) and electron impact ionization both followed by electron cascade. While the MPI process is self-sufficient, the electron impact ionization requires the presence of seed electrons in the laser focal region. The MPI process $\left[n h v+A \rightarrow A^{+}+\mathrm{e}+I_{\mathrm{P}}(A) ; n h v \geqslant I_{\mathrm{P}}(A)\right]$ involves the simultaneous absorption of a number of photons required to equal the IP of a gas. The ionization rate in MPI varies as $I_{\mathrm{W}}^{n}$ where $n$ is the number of photons needed to strip off an electron, which corresponds to the integer part of the quantity:

$$
n=\frac{I_{\mathrm{P}}+\varepsilon_{\mathrm{osc}}}{h v}+1,
$$

with $\varepsilon_{\text {osc }}$ the oscillation energy of a free electron in the alternating electric field. Within the classical microwave breakdown theory [36], a free electron oscillates in the alternating electric field $E$ of the laser electromagnetic wave (with frequency $\omega$ and wavelength $\lambda$ ), and its oscillation energy,

$$
\begin{aligned}
\varepsilon_{\mathrm{osc}}[\mathrm{eV}] & =\frac{e^{2} E^{2}}{4 m \omega^{2}}=\frac{e^{2}}{4 m \pi c^{3}} I_{\mathrm{W}} \lambda^{2} \\
& =4.67 \times 10^{-14} I_{\mathrm{W}}\left[\mathrm{W} \mathrm{cm}{ }^{-2}\right] \lambda^{2}[\mu \mathrm{m}],
\end{aligned}
$$

remains constant. On the other hand, MPI is relatively improbable for oxygen atoms or molecules in the ground state $\left[\mathrm{O}\left(2 \mathrm{~s}^{2} 2 \mathrm{p}^{4}{ }^{3} \mathrm{P}_{2}\right)\right.$ and $\left.\mathrm{O}_{2}\left(X^{3} \Sigma_{\mathrm{g}}^{-}\right)\right]$, since their high IPs (13.618 eV and $12.070 \mathrm{eV}$, respectively) mean that 117 photons 

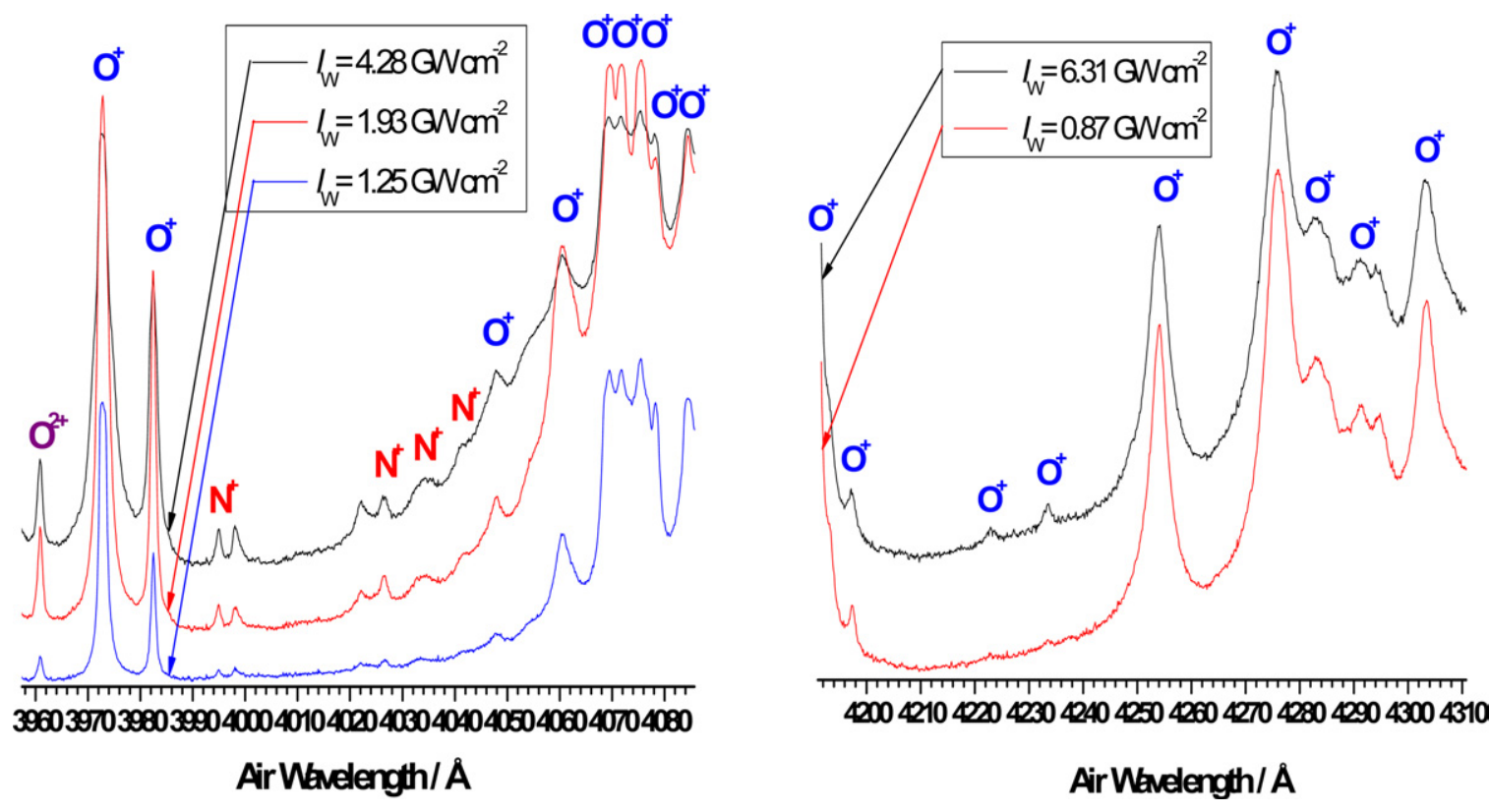

Figure 8. $(a)-(b)$ High-resolution LIB in oxygen (at a pressure of $48.8 \mathrm{kPa}$ ) emission spectra observed in the (a) $3957-4085 \AA$ and (b) 4195-4310 $\AA$ regions, excited by two TEA- $\mathrm{CO}_{2}$ laser wavelengths at $10.591 \mu \mathrm{m}$ as a function of the laser power density.

and 104 photons $(\lambda=10.591 \mu \mathrm{m})$, respectively, are required for these processes. The probability of MPI $W_{\text {MPI }}$, by absorbing simultaneously $n$ laser photons to strip off an electron, is expressed by the classical formula $[37,38]$

$$
\begin{aligned}
W_{\mathrm{MPI}}\left[\mathrm{s}^{-1}\right] \cong & \omega n^{3 / 2}\left(1.36 \frac{\varepsilon_{\mathrm{osc}}}{I_{\mathrm{P}}}\right)^{n} \\
= & 1.88 \times 10^{15} \lambda^{2 n-1}[\mu \mathrm{m}] n^{3 / 2} \\
& \times\left\{\frac{6.35 \times 10^{-14} I_{\mathrm{W}}\left[\mathrm{W} \mathrm{cm} \mathrm{cm}^{-2}\right]}{I_{\mathrm{P}}[\mathrm{eV}]}\right\}^{n} .
\end{aligned}
$$

Besides, the probability of simultaneous absorption of photons decreases with the number of photons $n$ necessary to cause ionization. In general, this probability is $W_{\mathrm{MPI}} \propto \Phi_{\mathrm{W}}^{n} \propto$ $F_{E}^{2 n}$. Calculations of MPI probability for $\mathrm{O}_{2}$ give $W_{\text {MPI }}=$ $4.6 \times 10^{-236} \mathrm{~s}^{-1}$ for the $\mathrm{CO}_{2}$ laser at $\lambda=10.591 \mu \mathrm{m}$ and $I_{\mathrm{W}}=6.31 \mathrm{GW} \mathrm{cm}^{-2}(n=104)$. For example, for a $193 \mathrm{~nm}$ (ArF) at the laser intensity $I_{\mathrm{W}}=1 \mathrm{GW} \mathrm{cm}^{-2}(n=2)$, the probability of MPI for $\mathrm{O}_{2}$ gives $W_{\text {MPI }}=1058 \mathrm{~s}^{-1}$. Electron impact ionization process consists of the absorption of light photon by free or quasifree electrons $\left(\mathrm{e}+n h v+A \rightarrow \mathrm{e}^{*}+A\right)$. These free or quasifree electrons can be produced by the effect of cosmic ray ionization (natural ionization), by means of MPI or by a breakdown induced in some gas impurity. In air at atmospheric pressure, the natural electron density is $\sim 10^{3} \mathrm{~cm}^{-3}$. These electrons in the focal volume gain sufficient energy, from the laser field through inverse bremsstrahlung collision with neutrals, to ionize oxygen atoms, molecules or ions by inelastic electron-particle collision resulting in two electrons of lower energy being available for starting the process again $\left\{\mathrm{e}^{*}\left[\varepsilon^{3} I_{\mathrm{P}}(A)\right]+A \rightarrow A^{+}+2 \mathrm{e} ; \mathrm{e}^{*}\left[\varepsilon^{3} I_{\mathrm{P}}\left(A^{+}\right)\right]+\right.$ $A^{+} \rightarrow A^{2+}+2 \mathrm{e}$, etc $\}$. Electron impact ionization is most important for the longer wavelengths used in this work $(\lambda=$ 9.621 and $10.591 \mu \mathrm{m})$. The observed ionic emissions are best explained by an electron impact ionization mechanism. The

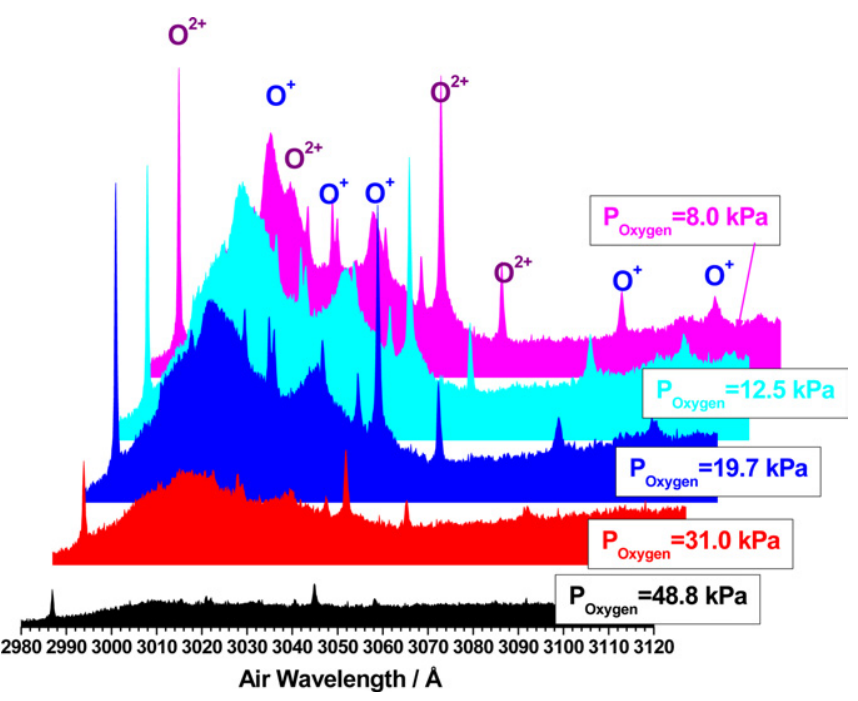

Figure 9. High-resolution LIB emission spectra at various oxygen pressures, excited by the TEA-CO $\mathrm{CO}_{2}$ laser $(10.591 \mu \mathrm{m})$ at a power density of $6.31 \mathrm{GW} \mathrm{cm}^{-2}$.

main role for MPI in our experimental conditions is to create seed electrons in the plasma plume.

On the other hand, we have made experimental measurements of breakdown threshold laser intensities of oxygen. The minimum optical laser power density required to form a plasma is called the breakdown threshold. The threshold power density is dependent on the kind of laser, laser wavelength, pulse length, beam size of the focal volume and gas pressure. Breakdown thresholds of solids and liquids are usually lower than for gases. Several LIB threshold measurements have been reported for oxygen [22-27] using $\mathrm{CO}_{2}$ [22] and Nd:YAG [23-27] lasers. The breakdown threshold was defined as the laser energy at which the gas would break down on more than $50 \%$ of the laser shots. 


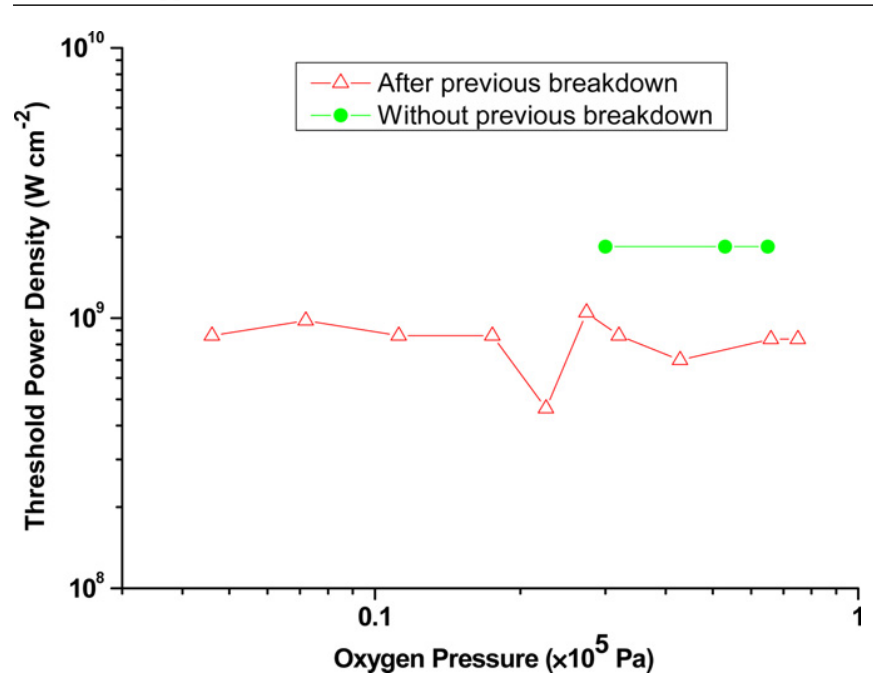

Figure 10. Experimental laser-induced breakdown thresholds in oxygen for different pressures. Solid circle: without previous breakdown; open triangle: after previous breakdown.

In the experimental section we explain the two methods that have been used to measure the breakdown threshold of oxygen. Some authors induce the breakdown at a pressure over the desired value, later the pressure is lowered and the energy adjusted until the breakdown begins with some probability, usually around $50 \%[26,27]$. This method is similar to inducing the breakdown with energy in excess and to attenuating the laser until the spark disappears. In these cases it could be that initial free electrons have been produced by previous breakdowns and they are the seeds of the avalanche process. Another way to induce the breakdown is to fix the pressure and to gradually increase the energy until a visible spark is observed around the focal region at the determined number of laser pulses [22]. In this last method the obtained threshold value is normally higher. We have used both methods to measure the oxygen breakdown at several pressures $(\lambda=10.591 \mu \mathrm{m})$ and the results are shown in figure 10. As can be seen from figure 10, if a breakdown has been previously induced in the gas (open triangles), the pressure range to induce the breakdown is bigger and the power density is approximately half than when no previous breakdown is induced (solid circles). The number of free electrons is higher in conditions of previous breakdown than in the case of no previous breakdown, lowering the threshold for the plasma initiation. This behaviour has been observed by different authors. Dewhurst [23] reported a decrease in the breakdown threshold intensity from $\sim 1.8 \times 10^{14}$ to $\sim 1.2 \times 10^{14} \mathrm{~W} \mathrm{~cm}^{-2}$ at $0.69 \mu \mathrm{m}$ and $\sim 5.5 \times 10^{13}$ to $\sim 1 \times$ $10^{13} \mathrm{~W} \mathrm{~cm}^{-2}$ at $0.53 \mu \mathrm{m}$ for oxygen pressures between $\sim 200$ and $~ 8000$ Torr. Chang et al [22] reported for several gases $\left(\mathrm{O}_{2}, \mathrm{~N}_{2}\right.$, air, $\mathrm{He}$ and $\left.\mathrm{Ne}\right)$ that the threshold power density decreases as the pressure increases and that it decreases as the focal volume increases. They reported a slow decrease with a little minimum of the breakdown threshold intensity from $\sim 15 \times 10^{9}$ to $\sim 5 \times 10^{9} \mathrm{~W} \mathrm{~cm}^{-2}$ at a diffusion length of $\Lambda=1.6 \times 10^{-3} \mathrm{~cm}$ and from $\sim 3 \times 10^{9}$ to $\sim 2 \times 10^{9} \mathrm{~W} \mathrm{~cm}^{-2}$ at a diffusion length of $\Lambda=3.2 \times 10^{-3} \mathrm{~cm}$ for oxygen pressures between $\sim 200$ and $\sim 10000$ Torr. In this work, they used a
$\mathrm{CO}_{2}$ laser and the focal diameter range from $0.75 \times 10^{-2}$ to $3 \times 10^{-2} \mathrm{~cm}$. Phuoc [27] reported a decrease in the breakdown threshold intensity from $\sim 1 \times 10^{13}$ to $\sim 2 \times 10^{12} \mathrm{~W} \mathrm{~cm}^{-2}$ at $0.532 \mu \mathrm{m}$ and from $\sim 8 \times 10^{12}$ to $\sim 1 \times 10^{12} \mathrm{~W} \mathrm{~cm}^{-2}$ at $1.064 \mu \mathrm{m}$ for oxygen pressures between 150 and 3040 Torr. It is to be noted that we have obtained similar threshold power densities for $\mathrm{O}_{2}$ to those given by Chang [22], but lower values than those reported in [23-27]. This fact can be related in part to the used focal length $(24 \mathrm{~cm})$ and beam size in the focal region $\left(7.85 \times 10^{-3} \mathrm{~cm}^{2}\right)$ that is one order of magnitude, at least, higher than the values commonly used in the literature, favouring the probability of the existence of free electrons to seed the process and decreasing the threshold laser intensity due to the lack of diffusion losses.

It has been established [3-11] that the threshold photon flux density or equivalently the threshold power density for MPI varies with $P^{-1 / n}$, where $P$ is the gas pressure and $n$ is the number of simultaneously absorbed photons. Therefore, MPI predicts a very weak dependence of the threshold power density on pressure. The measurements of the breakdown threshold intensity variation with the oxygen pressure (figure 10) reveal the weak pressure dependence, a characteristic of MPI. Our investigations of the threshold power densities covered the pressure range $P<65 \mathrm{kPa}$ without previous breakdown and $P<75 \mathrm{kPa}$ after previous breakdown. This pressure range is relatively limited due to the characteristics of the employed pyrex gas cell. Presumably, we suppose that the oxygen breakdown occurs in the high pressure range taking into account the experimental observations reported by Chan et al [22], Dewhurst [23], Stricker and Parker [24], Gamal [25] and Phuoc [26,27]. Therefore, starting from our experimental observations and calculations, we can conclude that although the measurements of the breakdown threshold intensity variation with the oxygen pressure (figure 10) reveal the weak pressure dependence, a characteristic of MPI, our calculations of MPI probability for $\mathrm{O}_{2}$ give $W_{\mathrm{MPI}} \cong 0$ for the $\mathrm{CO}_{2}$ laser at $\lambda \cong 10 \mu \mathrm{m}$ and $I_{\mathrm{W}} \cong 6 \mathrm{GW} \mathrm{cm}^{-2}$. So we think that although the first electrons must appear via MPI or natural ionization, ionization by electron impact is the main mechanism responsible for the breakdown in molecular oxygen.

The condition for optical breakdown is taken to occur when the number density of the induced electrons equals the critical density for the laser wavelength. The critical plasma density $n_{\mathrm{e}}^{\text {crit }}\left[\mathrm{cm}^{-3}\right]=m_{\mathrm{e}} \omega^{2} / 4 \pi e^{2} \cong 1.1 \times 10^{21} / \lambda^{2}[\mu \mathrm{m}]$ $\left(n_{\mathrm{e}}^{\text {crit }} \cong 10^{19} \mathrm{~cm}^{-3}\right.$ for $\mathrm{CO}_{2}$ laser) is the density where the electron plasma frequency equals the laser frequency. When the electron density exceeds the critical density the sample is not transparent any more. Energetic electrons produce excited species through impact excitation, dissociation and ionization of $\mathrm{O}_{2}$ molecules. The initial formed plasma expands within the space and supplies the spark gap with sufficient electrons, ionizing the gas and drastically reducing its electrical resistance; therefore, the breakdown occurs at electric fields less than the self-breakdown electric field of the threshold. The plasma is confined within a high-density shell of neutral oxygen gas that traps ionizing radiation in a region of low $E / N$ (where $E$ is the applied electric field divided by the total gas 
mixture density $N$ ); therefore inhibiting further expansion by non-hydrodynamic means electron avalanche. The reduced electric field $E / N$ (or equivalently $E / P$ where $P$ is the pressure; $P=N k_{\mathrm{B}} T$ ) gives an idea about the average energy that an electron possesses in plasma. The greater the value of $E / N$ (higher $E$ and/or lower $N$ ) the further apart is the average energy of the electrons from oxygen molecules. The non-equilibrium highly transient plasma (or plasma during its formative non-equilibrium phase), which is generated in $E / N$ conditions and applied during its formative phase, allows radicals $\left(\mathrm{O}, \mathrm{O}^{+}\right.$and $\left.\mathrm{O}^{2+}\right)$ and other electronically excited species $\left(\mathrm{N}, \mathrm{N}^{+}\right.$and $\left.\mathrm{N}_{2}^{+}\right)$to be generated over a relatively large volume. Active radical generation is due to molecule dissociation and excitation by electrons in the laser electric field, radiation and more complex processes. The presence of radicals or excited molecules can effectively improve ignition conditions but the problem is at the level of the required laser power density. If the electron cascade processes and chain chemical reactions are realized, the production of even a small number of free electrons and radicals can lead to a large benefit in total reaction rates, as well as diminishing of the required laser power density input. The key for transient plasma is the high magnitude of the local reduced electric field $E / N$ that is associated at the laser focal volume which may produce electrons in energies of about $10-15 \mathrm{eV}$ or more and which increases the probability of molecular oxygen dissociation significantly. Energetic electrons with high kinetic energies can effectively generate reactive species, which can then rapidly initiate or accelerate chain reactions. Generally speaking, any type of excitation (dissociation, ionization, electron excitation or vibrational excitation) affects the reaction rates positively, improving ignition conditions. The electron impact process is effective for a high magnitude of the reduced electric field $E / N$.

\section{Conclusions}

Laser-induced breakdown generated by $\mathrm{CO}_{2}$ laser pulses $(\lambda=$ 9.621 and $10.591 \mu \mathrm{m} ; \tau_{\mathrm{FWHM}}=64 \mathrm{~ns}$; power densities ranging from 0.87 to $6.31 \mathrm{GW} \mathrm{cm}^{-2}$ ) in oxygen has been investigated by means of OES. The plasma produced in oxygen gas and pressures ranging from 4.6 to $75 \mathrm{kPa}$ has been measured and analysed. The strong emission observed in the plasma region is mainly due to the relaxation of excited atomic $\mathrm{O}$ and ionic fragments $\mathrm{O}^{+}$and $\mathrm{O}^{2+}$. Excitation temperatures of $31500 \pm 1600 \mathrm{~K}$ and $23000 \pm 3000 \mathrm{~K}$ were estimated by means of $\mathrm{O}^{2+}$ and $\mathrm{O}^{+}$ionic lines, respectively. Electron number densities of the order of $(3.5-16.5) \times 10^{16} \mathrm{~cm}^{-3}$ were deduced from the Stark broadening of several ionic $\mathrm{O}^{+}$lines. The characteristics of the spectral emission intensities from different species have been investigated as functions of the oxygen pressure and laser irradiance. Optical breakdown threshold intensities in $\mathrm{O}_{2}$ at $10.591 \mu \mathrm{m}$ have been determined. On the basis of our observations and calculations we propose that although the first electrons must appear via MPI of any molecular species present in the focal volume, or by natural ionization, electron impact is the main mechanism responsible for the breakdown in molecular oxygen.

\section{Acknowledgments}

The authors acknowledge the support received in part by the DGICYT (Spain) Projects: MEC: CTQ2007-60177/BQU and MEC: CTQ2008-05393/BQU for this research.

\section{References}

[1] Emsley J 2001 'Oxygen', Nature's Building Blocks: An A-Z Guide to the Elements (Oxford, UK: Oxford University Press) pp 297-304

[2] Maker P D, Terhune R W and Savage C M 1963 Proc. 3rd Int. Conf. Quantum Electronics (Paris: Dunod) vol 2 p 1559

[3] Zeldovich Y B and Raiser Y P 1965 Sov. Phys._JETP 21190

[4] Bebb H B and Gold A 1966 Multiphoton Ionization of Hydrogen and Rare Gas Atoms, Physics of Quantum Electronics ed P L Kelly et al (New York: McGraw-Hill)

[5] Demichelis C 1969 IEEE J. Quantum Electron. 5188

[6] Isenor N R and Richardson M C 1971 Appl. Phys. Lett. 18224

[7] Lyman J L and Jensen R J 1972 Chem. Phys. Lett. 13421

[8] Morgan C G 1975 Rep. Prog. Phys. 38621

[9] Cantrell C D, Freund S M and Lyman J L 1979 Laser Induced Chemical Reactions and Isotope Separation in Laser Handbook vol 3b ed M L Stitch (Amsterdam: North-Holland)

[10] Lyman J L, Quigley G P and Judd O P 1980 Multiple-Photon Excitation and Dissociation of Polyatomic Molecules ed C D Cantrell (Berlin: Springer)

[11] Hermann J and Floch T L 2004 J. Appl. Phys. 963084

[12] Bekefi G 1976 Principles of Laser Plasma (New York: Wiley )

[13] Radzienxki L J and Cremers D A 1989 Laser Induced Plasma and Applications (New York: Dekker)

[14] Majidi V and Joseph M R 1992 Crit. Rev. Anal. Chem. 23143

[15] Yueh F-Y, Singh J P and Zhang H 2000 Encyclopedia of Analytical Chemistry (Laser-lnduced Breakdown Spectroscopy, Elemental Analysis) ed R A Meyers (Chichester: Wiley )

[16] Cremers D A and Radziemski L J 2006 Handbook of Laser-Induced Breakdown Spectroscopy (Chichester: Wiley)

[17] Miziolek A W, Palleschi V and Schechter I 2006 Laser-Induced Breakdown Spectroscopy (Cambridge: Cambridge University Press)

[18] Pasquini C, Cortez J, Silva L M C and Gonzaga F B 2007 J. Braz. Chem. Soc. 18463

[19] Camacho J J, Poyato J M L, Díaz L and Santos M 2007 J. Phys. B: At. Mol. Opt. Phys. 404573

[20] Camacho J J, Poyato J M L, Díaz L and Santos M 2007 J. Appl. Phys. 102103302

[21] Camacho J J, Díaz L, Santos M, Reyman D and Poyato J M L 2008 J. Phys. D: Appl. Phys. 41105201

[22] Chan C H, Moody C D and McKnight W K 1973 J. Appl. Phys. 441179

[23] Dewhurst R J 1978 J. Phys. D: Appl. Phys. 11 L191

[24] Stricker J and Parker J G 1982 J. Appl. Phys. 53851

[25] Gamal Y E E-D 1988 J. Phys. D: Appl. Phys. 211117

[26] Phuoc T X 2000 Opt. Commun. 175419

[27] Phuoc T X and White C M 2000 Opt. Commun. 181353

[28] Nordstrom R J 1995 Appl. Spectrosc. 491490

[29] NIST Atomic Spectra Database online at http://physics. nist.gov/PhysRefData/ASD/index.html

[30] Martin W C and Zalubas R 1983 J. Phys. Chem. Ref. Data 12323

[31] Griem H R 1974 Spectral Line Broadening by Plasmas (New York: Academic) 
[32] Griem H R 1964 Plasma Spectroscopy (New York: McGraw-Hill)

[33] Griem H R 1997 Principles of Plasma Spectroscopy (Cambridge: Cambridge University Press)

[34] McWhirter R W P 1965 Plasma Diagnostic Techniques ed R H Huddlestone and S L Leonard (New York: Academic) chapter 5
[35] Sreckovic A, Dimitrijevic M S and Djenize S 2001 Astron Astrophys. 371354

[36] MacDonald A D 1966 Microwave Breakdown in Gases (New York: Wiley)

[37] Raizer Y P 1991 Gas Discharge Physics (Berlin: Springer)

[38] Luther-Davies B, Gamaly E G, Wang Y, Rode A and Tikhonchuk V T 1992 Sov. J. Quant. Electron. 22289 